\title{
Audio-Visual Prosody: Perception, Detection, and Synthesis of Prominence
}

\author{
Samer Al Moubayed, Jonas Beskow, Björn Granström, and David House \\ Center for Speech Technology, Royal Institute of Technology KTH, Stockholm, \\ Sweden \\ sameram@kth.se, beskow@kth.se, bjorn@speech.kth.se, davidh@kth.se \\ http://www.speech.kth.se
}

\begin{abstract}
In this chapter, we investigate the effects of facial prominence cues, in terms of gestures, when synthesized on animated talking heads. In the first study a speech intelligibility experiment is conducted, where speech quality is acoustically degraded, then the speech is presented to 12 subjects through a lip synchronized talking head carrying head-nods and eyebrow raising gestures. The experiment shows that perceiving visual prominence as gestures, synchronized with the auditory prominence, significantly increases speech intelligibility compared to when these gestures are randomly added to speech.

We also present a study examining the perception of the behavior of the talking heads when gestures are added at pitch movements. Using eyegaze tracking technology and questionnaires for 10 moderately hearing impaired subjects, the results of the gaze data show that users look at the face in a similar fashion to when they look at a natural face when gestures are coupled with pitch movements opposed to when the face carries no gestures. From the questionnaires, the results also show that these gestures significantly increase the naturalness and helpfulness of the talking head.
\end{abstract}

Keywords: visual prosody, prominence, stress, multimodal, gaze, headnod, eyebrows, visual synthesis, talking heads

\section{Introduction}

There is currently considerable interest in developing animated agents to exploit the inherently multimodal nature of speech communication. As animation becomes more sophisticated in terms of visual realism, the demand for naturalness in speech and gesture coordination increases.

It has long been recognized that visual speech information is important for speech perception [1] [2]. There has been an increasing interest in the verbal and non-verbal interaction between the visual and the acoustic modalities from production and perception perspectives. Studies have reported possible correlations between acoustic prosody and certain facial movements. In [3] , correlation between $\mathrm{f} 0$ and eye-brows movements is discussed. In [4], correlations between f0 
movements and head-movements dimensions are reported and such movements are found to increase speech-in-noise intelligibility. Such coupling of movements in the acoustic and the visual modalities is usually highly variable, but an understanding of the redundancy of information in these two modalities can greatly help in developing audio-visual human-human and human-machine interfaces to guarantee a maximum amount of interaction. More recently, the contribution of facial prosody to the perception of the auditory signal has been investigated. In [4] it was shown that head movements can be used to enhance speech perception by providing information about the acoustic prosodic counterpart. In [5] and more recently in [6], it is shown that even these movements at the top of the head can aid speech comprehension. Moreover, people can highly discriminate the acoustic prosody of an utterance only by looking at a video showing the top of the head. All these studies suggest a highly shared production and perception of prosody between the acoustic and the facial movements, while knowledge on how to quantize this strong relation, and on how to deploy it in systems is still highly greatly lacking.

\section{Audio Visual Prominence}

\subsection{Acoustic Prominence}

Prominence is traditionally defined as when a linguistic segment (a syllable, a word or a phrase) stands out of its context, as defined by [7], others use prominence as the perceptual salience of a linguistic unit [8].

Since the studies in this work are done in Swedish, we present an overview of the acoustic correlates to prominence in the Swedish language. In Swedish, prominence is often categorized with three terms corresponding to increasing levels of prominence: stressed, accented and focused. Research has reported that the most consistent acoustic correlate of stress in Swedish is segmental duration [9]. In addition, overall intensity differences have also been studied among the correlates of stress, although these differences may not be as consistent as the durational differences [9]. As to accented syllables, according to the Swedish intonation model in [10], the most apparent acoustic difference for accented from an unaccented foot is the presence of an f0 fall, referred to as a word accent fall. Thus, accent as a higher prominence level than just stress is signaled mainly by f0, although an accented foot is usually also longer than an unaccented one [11]. Finally, in focal accent, which is the highest level of prominence, the primary acoustic correlates for distinguishing 'focused' from 'accented' words is a tonal one - a focal accent or a sentence accent rise following the word accent fall [10]. However, this f0 movement is usually accompanied by an increased duration of the word in focus, [12], and by moderate increases in overall intensity [13]. In our studies, we deal with prominence as a perceptual continuum, which is separate from its underlying semantic function. Looking at prominence from this stand has been suggested and used previously in [14]. 


\subsection{Visual Prominence}

Recently, there has been an increasing focus on the relation between the visual modality (the face) and acoustic prominence [15]. In [16], results on Swedish showed that in all expressive production modes, words which are produced with a focal accent exhibit greater variation in the facial parameters movements (articulators, eyebrows, head, etc.) than when the word is in a non-focused position. In [17], eyebrows movements and head nods, added to a talking head, are found to be a powerful cue to enforce the perception of prominence. In [18] and [19], an investigation on the interaction between the acoustic and the visual cues of prominence is conducted, the results of this study suggest that, during production, when a word is produced with a visual gesture, the word is also produced with a higher acoustic emphasis. The results also suggests that, from a perception perspective, when people see a visual gesture over a word, the acoustic perception of the word's prominence is increased. In [20], it is shown that visual movements do not only enhance the perception of focus, but can even decrease the reaction time in detecting focus; and in [21] it was shown that focus can be detected, not only through head or eyebrow movements, but even through lips movements.

\section{Acoustic Detection of Prominence}

The detection and quantification of prominence phenomena in speech can play an important role in many applications since it concerns the question of how speech is produced and how segments are contrasted, e.g. decoding in speech recognition, and hence can be used for syntactic parsing [22], speech comprehension [23], and more recently, research is focusing on the audio-visual function of prosody, for example, in [24] it was found that visual prominence can enhance speech comprehension if coupled with the acoustic. Hence, it is important for speech driven avatars to detect prominent segments in the speech signal to drive gestures.

Many studies have investigated the acoustic-prosodic cues to prominence on a syllable or on a word level, some using lexical and higher level linguistic information. This study focuses on estimating prominence using syllable based units, since in some applications, the segmental information words might not be available. This presents a theoretical challenge since such a method requires sufficient information about prominence inside the boundaries of the syllable unit. In addition, some prominence categories (levels) are perceptually based on word level, and hence reliably transcribed data on a syllable or vowel level is not available.

\subsection{Prominence Model}

In this work, we suggest a linear model for describing syllable prominence, and post integration of the estimated syllable prominence will estimate the underlying word prominence. Linear simple models have already shown good performance, as in [25] on English read and spontaneous speech. 
If $x$ is an observation sample from a random parameter $X$, we define the level of prominence of $x$ as:

$$
\operatorname{Prom}_{x}=1-f_{X}(x), f \in[0 . .1]
$$

Where $f_{x}$ is the normalized likelihood of $x$ in the random distribution of $X$. If $x$ is a feature vector of $n$ independent features $x=\left\{x_{1}, x_{2}, . ., x_{n}\right\}$, the prominence level of the observation vector $x$ is:

$$
\operatorname{Prom}_{x}=1 / n \sum_{i=1}^{n} w_{x_{i}} \operatorname{Prom}_{x_{i}}
$$

While $w_{x_{i}}$ is the weighting of $x_{i}$.

Since the data has only word level annotations, in the case when $x$ is a syllable level observation, we assume that the syllable with the higher prominence level in the word containing it provides the prominence of the word. In this case, the weights $w_{x_{i}}$ can be tuned to minimize the error of estimating the word prominence when using only syllable level features.

\subsection{Feature Set}

As shown in the Swedish prominence model in section 2.1, duration, loudness and F0 movements are major acoustic correlates of prominence. In this study, sets of either syllable level or word level features have been used. Features representing the syllables are taken from the syllable vowel, since vowels represent the nuclei and the acoustically stable part of the syllable: The set of features used include syllable duration, syllable loudness, and syllable F0 features including: average $\mathrm{F} 0$, average delta $\mathrm{F} 0$, and $\mathrm{F} 0$ range.

\subsection{Experiments and Results}

200 sentences were selected from a corpus containing news texts and literature, read by a professional Swedish actor. The corpus contains high-quality studio recordings for the purpose of voice creation for speech synthesis systems. The speech files from the 200 sentences dataset were phonetically aligned, and the acoustic features, were extracted over all the syllables. Half Gaussian distributions were estimated for each of the features, and the likelihood of each feature was calculated, and hence the prominence level per features. The data in all the tests was split into 4 cross validation folds giving $25 \%$ test set size. The baseline in this study was simply taken as the average word prominence for the whole dataset. This gives a fixed prominence value for all the words in the test set.

The weights were estimated by assuming that the syllable which has the highest averaged weighted prominence holds the underlying word prominence, in this case, the weights were optimized using a grid-search optimization to minimize the error on this post-integration function.

The $100 \%$ percentage accuracies for estimating word prominence for syllable features are presented in Figure 1, By looking at the results, it is shown that 


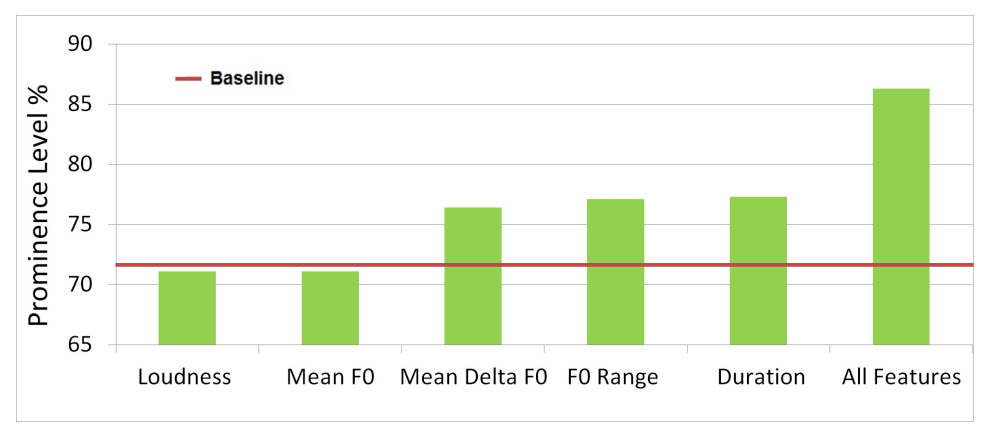

Fig. 1. The prominence detection percentage accuracies for single and multiple features against the baseline. The baseline gives a fixed value of the average prominence of all the words in the dataset

loudness, and mean F0 are unable to increase the accuracies beyond the baseline; it is good to mention that the distribution of the prominence values per word is highly unbalanced where most words are not prominent, and hence this simple baseline gave an accuracy of $72 \%$. Nonetheless, syllable F0 range performed individually better, and the performance was increased for the syllable duration. The best performance of $86 \%$ was reached using all the syllable features combined, with optimized weights equal to: Loudness: 0.13 , Duration: 0.52 , delta F0: 0.21, F0: 0, and F0 range: 0.14.

As a result, this model allows for detecting the syllables with the highest prominence in an utterance, which then allows the addition of facial gestures on these syllables as visual correlates for prominence. The investigation of effects of such gestures is carried out in the following studies.

\section{Visual Prominence and Intelligibility}

As mentioned earlier, several studies support the strong relation between the auditory and the visual modalities in perceiving prominence. These studies though, only report that the perception of prominence as a non-verbal prosodic phenomenon is highly aided by certain visual movements, and hence, the visual modality supports the realization of the acoustic prominence which exists in the audio stream.

Since prominence is manifested differently depending on the linguistic segment underlying it, and perceiving prominence aids speech comprehension, an interesting study is whether perceiving cues of prominence through the visual modality can increase speech intelligibility when the acoustic prominence is degraded. And hence, visualizing prominence on the talking head will not only be useful to deliver the function of its acoustic counterpart, but also support speech perception of the acoustic signal.

To investigate such effects, a speech intelligibility experiments was conducted with the help of a lip synchronized animated talking head. 


\subsection{Method and Setup}

In this study, a lip-synchronized talking head was used as the medium for the visual modality; this allows for manipulating the visual modality and keeping the acoustic one unchanged. The talking head used has been shown to provide increased intelligibility in several previous studies [26],[27]. This experiment design deploys the approach of presenting human subjects with a vocoded speech signal, while the subjects are looking at a talking head [28].

40 semantically complete sentences, ranging in length from 6 and 10 words, were selected from the same database used for the detection experiments above.

The speech files of the 40 sentences were force-aligned using an HMM aligner [29] to guide the talking head lips movement generation procedure [30]. The audio was processed using a 4-channel noise excited vocoder [31] to reduce intelligibility. The number of channels was decided after a pilot test to ensure an intelligibility rate between $25 \%$ and $75 \%$, that is to avoid any floor or ceiling limit effects.

All the sentences were presented to subjects with an accompanying talking head. The first 10 sentences were presented without any facial gestures, as a training session, to eliminate any quick learning effect for the type of signal vocoding used. The 30 sentences left were divided into 6 groups; every group contained 5 sentences, with a balanced number of words in each group (3540 words). For each group, 6 different visual stimuli were generated (details in section 4.3). These groups were systematically permuted among 12 normal hearing subjects with a normal or corrected to normal vision, so that every subject listened to all 30 sentences, but with each group containing different visual stimuli. During the experiment, the sentences were randomized for every subject. The subjects had one chance to listen to the sentence, and report what they have perceived as text from the audio visual stimuli.

\subsection{Marking Prominence}

For the purpose of this study, the gestures which are included in the stimuli are fixed in length and amplitude. And since visual correlates to prominence must be synchronized with their acoustic counterpart, we decided to limit the size of the prominent segment from the whole word to its most prominent syllable, so the gestures are associated with prominent syllables not with words.

To establish that, a native Swedish speech expert had listened to all the 30 test sentences, and marked them temporally for prominence. In this study, the annotation of only one annotators was taken. By investigating the prominence markers, all sentences have received between 1 to 3 prominence marks, and the overall number of marks in the 30 sentences summed to 60 .

\subsection{Conditions}

Following is a detailed description of these variants. It is important to note that, when a sentence received gestures, the number of the gestures that this sentence received was identical in all the conditions (except for the control condition). 


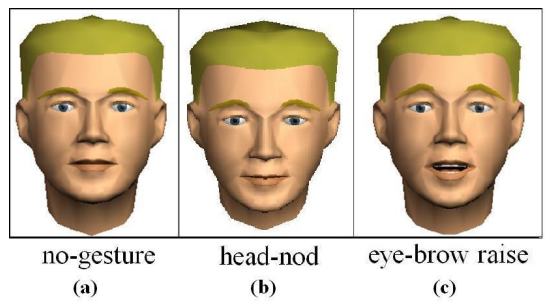

Fig. 2. Snapshots of the talking head in different gestural positions. (a) neutral parameters. (b) lower point of the head-nod gesture. (c) peak of the eyebrow raising gesture.

- No Gesture (N): The first condition is 'articulators-only' where the face is static, except for the lips-jaw area for the purpose of phonetic articulation. This condition is intended to be a control measurement for the rest of the conditions. Figure 2a displays the talking head in the neutral position.

- Prominence with Head-nods (PH): In this condition, a head-nod was synthesized in synchrony with the place of the prominence markers in each sentence. The design of the headnod gesture was near-arbitrary, consisting of subtle lowering and rising to the original location, the complete motion length was set to $350 \mathrm{~ms}$, which is an estimate of the average length of a stressed syllable in Swedish. Figure 2b shows the talking head at the lower turning point of the head nod.

- Prominence with Eyebrows Raise (PEB): The stimulus in this condition matches the one of the head-nod, except that the gesture in this stimulus is an eyebrow raising, with a matching design in length of trajectories as the head-nod gesture. Figure 2c shows the eyebrow raising gesture at its top turning point.

- Automatically detected prominence with Eyebrows (AP): In this condition, prominent syllables were automatically detected using the automatic prominence detection method explained earlier (section3), and eyebrow raising gesture was coupled with these syllables.

- Pitch Movement (PM): In this condition, eyebrow raise gestures were temporally placed over steep pitch movements. The advantage of this condition is that it is automatically detected, and so it can easily be used for real-time visual speech synchrony in talking agents.

- Random Eyebrows Raise (R): This conditions adds the eyebrow raising gesture randomly on syllables. This condition is made to investigate whether random gestures would benifit or hinder the perception of the acoustic signal.

\subsection{Analysis and Results}

A string based percentage correct word recognition scoring was manually applied to the answer of each of the sentences of the subjects, and the average recognition rate per sentence was then calculated. As a result, every condition received 60 samples $\left(12\right.$ subject $^{*} 5$ sentences per condition). 


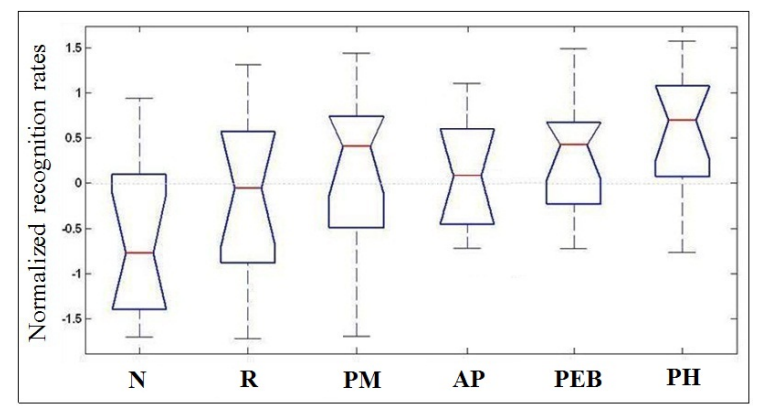

Fig. 3. Box plot of the within subject normalized $\%$ correct recognition over conditions.

An ANOVA with normalized recognition rates (Z-scored over subjects) as a dependent variable, and Condition (5 levels: No Gesture, Random, Pitch Movements, Prominance Head-nod, and Prominence EyeBrows raise) as an independent variable was applied. Subjects were not included as a factor since persubject z-score normalization was applied to effectively remove within-subject differences.

Condition gave a significant main effect: $\mathrm{F}(4,295)=4.4, p<.01$. An LSD post hoc test showed that the conditions: No-Gesture against Prominence Head-nods, No-Gesture against Prominence EyeBrows raise, No-Gesture against Pitch Movements, and Prominence Head-nods against Random eyebrows, are all different using a significance level of .05. It is interesting to notice that the only condition which had significant difference from the random condition $(\mathrm{R})$ was the head-nod $(\mathrm{PH})$ condition, which, in terms of mean recognition rate, had the highest value among all the other conditions (Figure 3). This can be explained by the fact that head-nods actually aid the perception of prominence more than eyebrows, and that more test subjects should be considered to reach a significant difference.

These results mainly indicate that adding head-nods or eyebrow raising on prominent syllables or accented syllables increases speech intelligibility compared to looking at the talking head without gestures at all. On the other hand, adding these gestures randomly over speech, does not increase intelligibility compared to not adding any gestures.

In this study nonetheless, the gestures employed are not optimal or identical to movements employed by humans, but it is still plausible to assume that these movements to some degree carry the same communicative information contained in human gestures.

Gestures and facial movements are a characteristic of audio-visual interaction, and they might have other roles which affect intelligibility and comprehension than indicating prominence. Which might be the reason why there was no significant difference between the random condition and the prominence and pitch movements eyebrows conditions.

Since these facial movements correlate with acoustic prominence, and as concluded in the previous tests, they support speech intelligibility, a natural question 
to these results is: how will these movements support the interaction between humans and talking heads and in what ways would they affect it.

To study such effects, a follow up study was conducted by using a talking head as a speech synchronized visual support for an audio-based story.

\section{$5 \quad$ Naturalness and Gaze Behavior}

To investigate the perceived non-verbal effect of synthesizing the gestures coupled with auditory prominence, an ecological experimental setup was designed by using the SynFace speech driven talking head [27] designed as a visual support for hard of hearing persons [32]. The talking head used in SynFace is the same talking head used in the previous experiment, but SynFace applies a real-time phoneme recognition on the input speech signal to drive the talking head, rather than forced alignment, as was the case in the previous experiment.

The experiment setup is based on presenting an audio novel (audio-book). The audio-book is presented to moderately hearing impaired subjects and the subjects have to evaluate the talking head in different conditions. During listening to the audiobook, the subjects' eye-gaze is tracked using a Tobii T $120^{1}$ eye-gaze tracker, which is integrated into the monitor on which the talking head was presented. At the end of listening to each of the audiobook conditions, the subjects were presented with a questionnaire form, Table 1 shows the questions included in the questionnaire form.

\subsection{Stimuli and Setup}

Subjects firstly had the option to choose one of a long list of audiobooks obtained from the Swedish Library of Audio-and Braille books ${ }^{2}$. The first 15 minutes of each of the audio-books chosen by the subjects were split into 3 parts, each of 5 minutes. The three parts are then randomly manipulated into three conditions: Only audio, audio and talking head (articulatory movements only), and audio and talking head (articulatory movements and gestures). Refer to section 5.2 for a detailed explanation on how the gestures are generated.

After generating the stimuli, 6-speakers stereo babble noise was added to the audio signal to reduce the understanding level of the audiobook. To decide the SNR level of the audio-book, a small speech-in-noise intelligibility experiment was conducted for each subject to calibrate the SNR level.

For the calibration experiments, 15 short Swedish everyday sentences were used, these sentences were between 7-9 syllables in length, and developed specifically for intelligibility testing, based on [2], and used in several previous intelligibility experiments (c.f.[26]), each of these sentences contains three keywords. The SNR was calibrated with a step of $1 \mathrm{~dB}$ starting initially at an SNR equal to OdB to reach an approximate $50 \%$ keyword intelligibility rate. After calibration,

\footnotetext{
${ }^{1}$ http://www $\cdot$ tobii.com/

${ }^{2}$ The Library of Audio-and Braille books: http://www.tpb.se
} 
the audio-book SNR level was changed to the calibrated value for each subject plus $2 \mathrm{~dB}$ to allow for an easier understanding of the signal (which in principle should be supported further by viewing the talking head).

A set of 10 moderately hearing impaired male subjects took part in the experiment, with an average age of 71.2 years old. Subjects were seated approximately $60 \mathrm{~cm}$ from the screen.

\subsection{Gesture Generation}

For all the audio-book parts, pitch movements were detected using the method described in section 4.3. Since the automatic detection function showed that it enhances speech intelligibility significantly when coupled with an eyebrow raising, this gives the possibility of automatically detecting pitch movements which allows for a direct implementation in talking heads.

As to what gestures to be synthesized, in [16], all facial parameters seemed to exhibit big variations under prominent words, and in the previous experiment, head-nods and eyebrow raising, have shown to enhance speech comprehension. In addition to these cues, eye blinks have been used as a correlate to stress, and used in talking heads as in [33] based on a model suggested by P. Ekman in [34]. Eye blinks are important biological movements, that have been suggested to synchronize with speech production [35]. They play an important role in the perception of natural facial movement, in speaking, reading and listening situations and are highly correlated with the cognitive state of the speaker [36].

To introduce variability to the facial movements in the talking head, headnods, eyebrow raising, and eye blinks are coupled with the automatically detected pitch movements. And in order to regulate the amount of gestures and avoid unnatural movement, a maximum of one gesture was allowed to be synthesized inside a moving window of 1 second, that is depending on the steepness the pitch movements detected inside this window (see 4.3). After deciding on the place where the gesture will be added, a uniform random function chooses one of the head-nods, eyebrow raising, or blinks to be added.

\subsection{Gaze Analysis}

The gaze tracking data of the audiobook sessions was collected for all 10 subjects, and divided into 2 parts for each subject: gaze for a talking head with gestures, and gaze for the talking head without gestures. The first test split the data into two Areas Of Interest (AOI): Face and Not Face, as displayed on the right in Figure 4. The second test split the data into three AOI: Mouth region, Eyes region, and Other. Other represents gaze movements outside the mouth and eyes regions. The left side of Figure 4 shows the location of these AOIs. Two types of visualizations are applied to present the gaze data, the first one is called a Gaze Plot which visualizes the fixations as circles and the saccades as lines between fixations, the size of the circles defines the length of the fixation, so the bigger the circle longer the fixation. The other type is called Gaze Heat Map where the 
face is cleared in regions where there is higher intensity of gaze and shadowed on less visited areas. Both visualizations are generated from the same data.
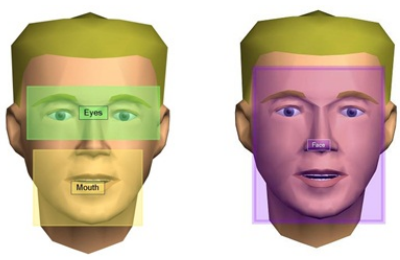

Fig. 4. Left: The eyes and mouth regions of the face. Right: The inside and outside regions of the face.

Figure 5 shows the Gaze Plot and Heat Map for all the subjects for the two SynFace versions (left: without gestures, right: with gestures). In this figure, it is clear that a wider area of the face was scanned by the subjects in the SynFace with gestures, specially around the eyes, this behavior is more similar to the normal eye-gaze behavior during audio-visual speech perception in noise that is reported in the literature [36] [37]. While in the talking head with no gestures condition (left), the subjects only focused on the mouth, which could be due to the understanding that information about the audio signal is only present in and around the lips and articulators. In the talking head with gestures condition, the subjects browsed the face at all time by fixations and saccades from the mouth to the eyes. It is also clear in the plot, that the saccades into external regions outside the face have been significantly lessened with the use of gestures in the face.

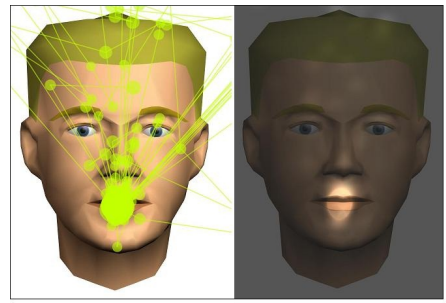

Without Gestures

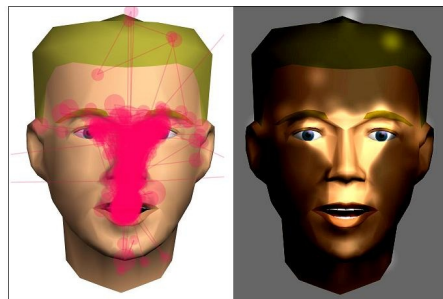

With Gestures

Fig. 5. Gaze tracking results for the ten subjects, showing gaze plot and heat map for both conditions (with and without gestures)

To measure the time the user spent looking at the face during listening to the audio-book, the sum of the fixation length is measured for the areas of interest for all subjects, and for both versions of the face. The left side of Figure 6 
presents the average percentage of time in and outside the face for both versions of the face. The figure shows that the time spent by the subjects looking at the face increased from $76 \%$ to $96 \%$ when gestures are present. And the right side of Figure 6 presents the average percentage of time on the defined regions of the eyes, mouth and the rest of the face. It is interesting to see that the eyes region has received gaze significantly more in time than the face with no gestures, and the area outside the face has received significantly less. This can be explained in that subjects were more engaged with the talking head so that the time spent not looking at the face was lessened when gestures were present. It is also interesting to see that the time spent looking at the mouth has lessened when gestures are present, this means that when gestures are generally present on the face, they draw the gaze away from the mouth. This, nonetheless, does not mean that the information extracted from the mouth region is reduced when gestures are synthesized since peripheral vision can still help perceiving lip movements even if the eyes are not fixated on the lips, as found in [38].
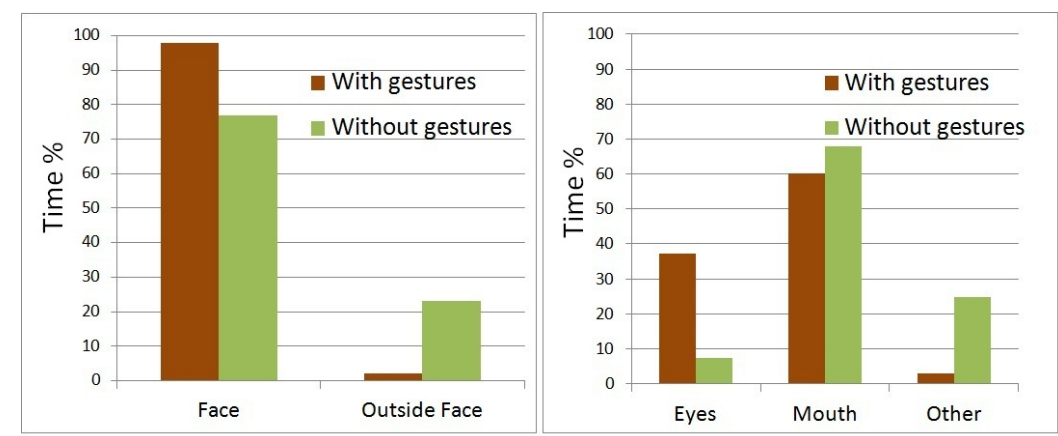

Fig. 6. The percentage of time spent inside and outside the Area Of Interest (AOI) in the two conditions.

\subsection{Questionnaires Analysis}

Looking at Table 1, the questions in the questionnaire target the subjective opinions of the test subjects after listening to the audio-book for each version of the face.

Figure 7 shows the mean and standard deviation of the difference between the ratings for the with gestures face and the no gestures face for all the questions, hence the 0 point represents the rating of the no gestures condition for each of the questions.

An ANOVA was performed with question rating and subject as a dependent variable, and condition ( with gestures, without gestures) as an independent variable on each of the questions. The results show a significant mean effect 
Table 1. The format of the questionnaire form presented to the subjects.

\begin{tabular}{ll}
\hline Question & Answers $[1 . .5]$ \\
\hline 1-How much did you understand? & nothing .. everything \\
2-How much effort did it take to understand? & no effort .. much effort \\
3-Did the face help? & not at all .. helped me a lot \\
4-How much did you watch the face? & not at all .. all the time \\
5-How natural was the face? & unnatural .. very natural \\
\hline
\end{tabular}

for the Helpfulness (third question), Watching Duration (fourth question) and Naturalness (fifth question), all at a significance level of .05.

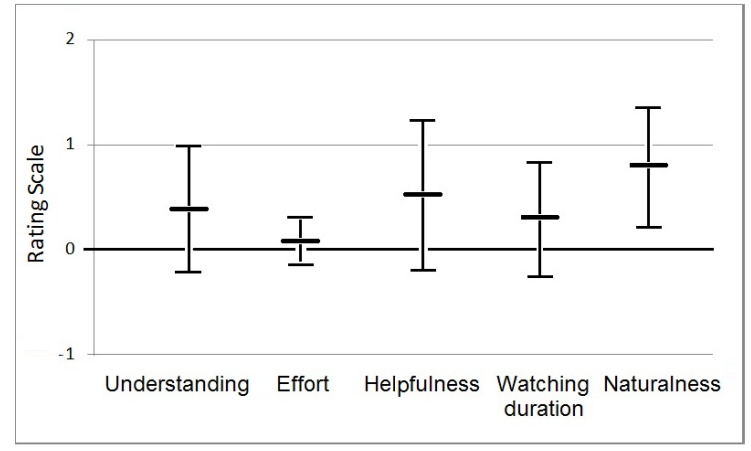

Fig. 7. A plot of the mean and standard deviation of the questionnaire ratings for the face with gestures conditions compared to 0 as the answer for the face with no gestures.

The results of the questionnaires show that subjects spend more time watching the face when gestures are synthesized. This is Consistent with the results from the gaze analysis. Additionally, the gestures over pitch movements result in a animated talking face which is significantly more natural, and subjectively presents a more understandable face than the face with no gestures.

\section{Discussions and Remarks}

The results from the intelligibility experiment indicate that when head-nods and eyebrow raise gestures are visualized during prominent syllables, they can aid speech perception. On the other hand, the results do not present a strong evidence on whether visualizing them during non-prominent syllables may hinder or aid perception.

The speech signal in this test was vocoded, and no pitch information was available to the listener, which may result in a decreased amount of information about the syllabic boundaries in the signal. The visualization of gestures 
then might be a possible source of voicing information (which aligns with the significant increase of the condition with pitch movements (PM) over the the condition with no gestures $(\mathrm{N})$. We believe that an important function of gestures is temporal resolution and segmentation of the acoustic stream. If gestures during prominence provide information about the syllable boundaries of the prominent syllable, this can, in addition to providing semantic and pragmatic information about the sentence provide segmental information (syllabification) of the underlying acoustic stream.

In Japanese [39], it was found that pitch movement can help in the selection of word candidates. In Swedish, syllables in words are contrasted through lexical stress. It is possible that visual prominence, aligned with prominent syllables, can provide information about the segmental structure of the underlying word, and hence help in shortening the candidate list for the mental lexicon access.

It was shown before that the perception of head movements can increase speech intelligibility [4], and that the motion at only the top of the head can do the same but more reliably in expressive sentences [5]. These studies have used, as stimuli, human recordings of head movements, and hence could not provide quantified information on when these movements communicated their effect. The present experiment, in addition to showing that visual cues of acoustic prominence can aid speech intelligibility, also quantifies this effect through the use of a minimal model of fixed head nods and eye-brows raise movements on well-defined instants in time.

In [40], there is neuro-physiological evidence that matching visual information speeds up the neural processing of auditory information, although where and when the audio-visual representation of the audio-visual signal is created remains unsolved, it is evident that perceiving visual information increases the processing of the auditory stream and hence provides more temporal resolution; from this view, visualizing prominence may also provide information about speech rhythm and syllables boundaries of the underlying linguistic segment.

Going from the verbal effects of visual prominence to the interaction effects, we conducted the audio-book experiment. It has long been recognized now that there is much information present in the face, in addition to the articulators, which provide information about the speech. Animating this visual information makes virtual characters more human-like by exhibiting more variant, complex and human like behavior. In this study, we demonstrated that when gestures are coupled with prominence in acoustically degraded stimuli, the gaze behavior of subjects significantly changed into patterns which closely resemble those used when looking at a real human face; moreover, this also lessened the time the subjects spent looking away from the face. This suggests that the subjects' engagement with the talking head is increased when these gestures are visualized, while these gestures added to the naturalness and helpfulness of the talking face. Many subjects have reported after the experiments that they did not notice that the talking head had embedded gestures in it, which might be a possible indication that the perception of these gestures is realized on a subconscious level; while other subjects have reported that they were excited by perceiving gestures 
manifested by the face, and that was an indication of some sort of intelligence of the virtual agent.

\section{Conclusion}

We have investigated whether visual correlates to prominence can increase speech intelligibility. The experimental setup in this study used a lip synchronized talking head. By conducting an audio-visual speech intelligibility test, using facial gestures during prominent syllables, it was found that head-nods and eyebrow raising gestures significantly increased recognition rate. These results reveal new evidence that information about the verbal message is carried by non-verbal visual gestures. The experiment also provides a possibility to deploy these gestures in talking heads which would provide a medium for audio-visual speech perception. We also investigated the effects of synthesizing these gestures over syllables with steep pitch movements on the eye-gaze behavior and on the subjective opinions of moderately hearing impaired subjects; the results show that users' eye gaze extends from only the mouth region in the articulatory only face to the eyes and mouth in the gestural face. In addition to that, the subjects' opinions through questionnaires show an increase in intelligibility of the face when these gestures are added.

This result opens the possibility for talking heads to use visual correlates to prominence to support visual speech perception and aid the communication of prominence through the facial modality. An important application is to synthesize these cues in multimodal speech synthesis and speech synchrony systems, which requires developing real-time automatic prominence detection systems.

While the experiments show the roles of gestures synchronized with prominence, the question of how exactly users parse this audio-visual information and enhance their speech perception using it still need to be investigated. Another interesting question is the study of the rate, design and amplitude of these gestures, and to find what is optimal to provide a more natural and useful animated systems.

Acknowledgments. This work was carried out at the Center for Speech Technology, a competence center at KTH supported by the Swedish research council project \#2005-3488. Part of this work is supported by the Swedish national Graduate School for Language Technology GSLT

\section{References}

1. H. McGurk and J. MacDonald, :Hearing lips and seeing voices. vol 264, p.746-748, (1976)

2. Q. Summerfield, :Lipreading and audio-visual speech perception. Philosophical Transactions: Biological Sciences, vol. 335, no. 1273, pp. 71-78, (1992) 
3. C. Cave, I. Guaïtella, R. Bertrand, S. Santi, F. Harlay, and R. Espesser, :About the relationship between eyebrow movements and Fo variations. Proc of the Fourth International Conference on Spoken Language, vol. 4, (1996)

4. K. Munhall, J. Jones, D. Callan, T. Kuratate, and E. Vatikiotis-Bateson, :Head Movement Improves Auditory Speech Perception Psychological Science, vol. 15, no. 2 , pp. 133-137, (2004)

5. C. Davis and J. Kim, :Audio-visual speech perception off the top of the head. Cognition, vol. 100, no. 3, pp. 21-31, (2006)

6. E. Cvejic, J. Kim, and C. Davis, :Prosody off the top of the head: Prosodic contrasts can be discriminated by head motion. Speech Communication, (2010)

7. J. Terken and D. Hermes:The perception of prosodic prominence, in Prosody: Theory and Experiment. Studies Presented to Gösta Bruce. pp. 89-127 , (2000)

8. B. Streefkerk, L. Pols, and L. Bosch, :Acoustical features as predictors for prominence in read aloud Dutch sentences used in ANN's. in Sixth European Conference on Speech Communication and Technology. Citeseer, (1999)

9. G. Fant, A. Kruckenberg, and L. Nord, :Durational correlates of stress in Swedish, French, and English. Journal of phonetics, vol. 19, no. 3-4, pp. 351-365, (1991)

10. G. Bruce, Swedish word accents in sentence perspective. LiberLäromedel/Gleerup, (1977)

11. C. Gussenhoven and G. Bruce, :Word prosody and intonation.Empirical Approaches to Language Typology. pp. 233-272, (1999)

12. M. Heldner and E. Strangert, :Temporal effects of focus in Swedish. Journal of Phonetics, vol. 29, no. 3, pp. 329-361, (2001)

13. G. Fant, A. Kruckenberg, J. Liljencrants, and S. Hertegård, :Acoustic phonetic studies of prominence in Swedish. KTH TMH-QPSR, vol. 2, no. 3, p. 2000, (2000)

14. G. Fant and A. Kruckenberg, :Notes on stress and word accent in Swedish. Proceedings of the International Symposium on Prosody, Sept 18 1994, Yokohama, pp. $2-3,(1994)$

15. B. Granström and D. House, :Audiovisual representation of prosody in expressive speech communication. Speech Communication, vol. 46, no. 3-4, pp. 473-484, (2005)

16. J. Beskow, B. Granström, and D. House, :Visual correlates to prominence in several expressive modes. Proc of the Ninth International Conference on Spoken Language Processing, (2006)

17. D. House, J. Beskow, and B. Granström, :Timing and interaction of visual cues for prominence in audiovisual speech perception. Proc of the Seventh European Conference on Speech Communication and Technology, (2001)

18. M. Swerts and E. Krahmer, :The importance of different facial areas for signalling visual prominence. Proc of the Ninth International Conference on Spoken Language Processing, (2006)

19. E. Krahmer and M. Swerts, :The effects of visual beats on prosodic prominence: Acoustic analyses, auditory perception and visual perception. Journal of Memory and Language, vol. 57, no. 3, pp. 396-414, (2007)

20. M. Dohen and H. Lœvenbruck, :Interaction of audition and vision for the perception of prosodic contrastive focus. Language and Speech, vol. 52, no. 2-3, p. 177, (2009)

21. M. Dohen, H. Lcevenbruck, and H. Hill, :Recognizing Prosody from the Lips: Is It Possible to Extract Prosodic Focus. Visual Speech Recognition: Lip Segmentation and Mapping, p. 416, (2009)

22. D. Wang and S. Narayanan, :An acoustic measure for word prominence in spontaneous speech. IEEE Transactions on Audio, Speech, and Language Processing, vol. 15 , no. 2, pp. 690-701, (2007) 
23. M. Grice and M. Savino, :Can pitch accent type convey information status in yes-no questions. Proc of the Workshop Sponsored by the Association for Computational Linguistics, pp. 29-38, (1997)

24. S. Al Moubayed and J. Beskow:Effects of visual prominence cues on speech intelligibility.Proceedings of the International Conference on Auditory Visual Speech Processing AVSP'09, vol. 15, p. 16, (2009)

25. F. Tamburini:Prosodic prominence detection in speech.Proceedings of the Seventh International Symposium on Signal Processing and Its Applications, vol. 1, (2003)

26. E. Agelfors, J. Beskow, M. Dahlquist, B. Granström, M. Lundeberg, K.-E. Spens, and T. Öhman, :Synthetic faces as a lipreading support. Proceedings of ICSLP'98, (1998)

27. G. Salvi, J. Beskow, S. Al Moubayed, and B. Granström, :Synface - speech-driven facial animation for virtual speech-reading support. Journal on Audio, Speech and Music Processing, (2009)

28. J. Beskow, :Rule-based visual speech synthesis. Proc of the Fourth European Conference on Speech Communication and Technology, (1995)

29. K. Sjölander, :An HMM-based system for automatic segmentation and alignment of speech. Proceedings of Fonetik, pp. 93-96, (2003)

30. J. Beskow, :Trainable articulatory control models for visual speech synthesis. International Journal of Speech Technology, vol. 7, no. 4, pp. 335-349, (2004)

31. R. Shannon, F. Zeng, V. Kamath, J. Wygonski, and M. Ekelid, :Speech recognition with primarily temporal cues. Science, vol. 270, no. 5234, p. 303, (1995)

32. S. Al Moubayed, J. Beskow, A.-M. Oster, G. Salvi, B. Granström, N. van Son, and E. Ormel:Virtual speech reading support for hard of hearing in a domestic multi-media setting. Proceedings of Interspeech09, (2009).

33. I. Poggi, C. Pelachaud, and F. De Rosisc, :Eye communication in a conversational 3D synthetic agent. AI communications, vol. 13, no. 3, pp. 169-181, (2000)

34. P. Ekman, :About brows: Emotional and conversational signals. Human ethology: Claims and limits of a new discipline: contributions to the Colloquium, pp. 169-248, (1979)

35. J. Cassell, C. Pelachaud, N. Badler, M. Steedman, B. Achorn, T. Becket, B. Douville, S. Prevost, and M. Stone, :Animated conversation: rule-based generation of facial expression, gesture \& spoken intonation for multiple conversational agents. Proceedings of the 21st annual conference on Computer graphics and interactive techniques, pp. 413-420, (1994)

36. S. Raidt, G. Bailly, and F. Elisei, :Analyzing and modeling gaze during face-to-face interaction. Proceedings of the International Conference on Auditory-Visual Speech Processing (AVSP07), (2007)

37. E. Vatikiotis-Bateson, I. Eigsti, S. Yano, and K. Munhall, :Eye movement of perceivers during audiovisual speech perception. Perception and Psychophysics, vol. 60, no. 6, pp. 926-940, (1998)

38. M. Paré, R. Richler, H. TEN, and K. Munhall, :Gaze behavior in audiovisual speech perception: The influence of ocular fixations on the McGurk effect. Perception \& psychophysics, vol. 65 , no. 4, p. 553, (2003)

39. A. Cutler and T. Otake, :Pitch accent in spoken-word recognition in Japanese. The Journal of the Acoustical Society of America, vol. 105, p. 1877, (1999)

40. V. van Wassenhove, K. Grant, and D. Poeppel, :Visual speech speeds up the neural processing of auditory speech. Proceedings of the National Academy of Sciences, vol. 102, no. 4, p. 1181, (2005) 